\title{
NeuroTechnique
}

\section{Validation of Internal Control Genes for Expression Studies: Effects of the Neurotrophin BDNF on Hippocampal Neurons}

\author{
Ana Rita A. Santos and Carlos B. Duarte ${ }^{\star}$ \\ Center for Neuroscience and Cell Biology, Department of Zoology, University of Coimbra, \\ Coimbra, Portugal
}

The stability of expression of an internal control is required for accurate and reliable normalization in quantitative real-time reverse transcription-polymerase chain reaction (qRT-PCR) experiments. However, expression of commonly used reference genes can be regulated under specific experimental conditions, particularly in response to stimuli that exert multiple effects on gene expression. The neurotrophin brain-derived neurotrophic factor (BDNF) regulates gene expression through activation of multiple signaling cascades, and we have conducted an expression study for the proper validation of internal control genes in BDNF-stimulated cultured hippocampal neurons. geNorm and NormFinder were applied to eight potential genes to identify the most stable genes to be used in the relative quantification of the effects of BDNF on gene expression. Our data show that Tbp (TATA box binding protein), Ppia (peptidylprolyl isomerase A), Pgk1 (phosphoglycerate kinase 1), and Hprt1 (hypoxanthine guanine phosphoribosyl transferase I) are the most stable genes under the experimental conditions used, contrasting with Tuba1 (tubulin alpha1-A chain) and Gapdh (glyceraldehydes-3-phosphate dehydrogenase), two genes widely used as control genes, which showed an unstable expression in hippocampal neurons stimulated with BDNF. Analysis of the BDNF-induced changes in expression of Sars, Tufm, and Egr3 by using different sets of control genes showed distinct results, with a combination of three to four of the genes Tbp/Ppia/Pgk1/ Hprt1 providing the most consistent results. Our data reinforce the need for proper validation of the internal control genes for an accurate quantification of qRT-PCR results, particularly when analyzing cellular responses to agents (e.g., neurotrophins) that cause multiple changes in gene expression. ๑ 2008 Wiley-Liss, Inc.

Key words: brain-derived neurotrophic factor; quantitative real-time reverse transcription-PCR; internal control gene; normalization
Neurotrophins play important roles in the regulation of axonal and dendritic growth and guidance, synaptic structure and connections, short- and long-term changes in synaptic activity, and neuronal survival and neuroprotection (Huang and Reichardt, 2001; Chao, 2003; Almeida et al., 2005; Schweigreiter, 2006; Caldeira et al., 2007; Manadas et al., 2007; Carvalho et al., 2008). Furthermore, neurotrophins contribute to glial cell development and survival (Althaus and RichterLandsberg, 2000; Schweigreiter, 2006; Manadas et al., 2007). Brain-derived neurotrophic factor (BDNF) is the most widely distributed neurotrophin in the adult brain, acting through the activation of the Trk (tropomyosin-related kinase) B and p75 neurotrophin receptors (Chao, 2003; Reichardt, 2006; Manadas et al., 2007). Binding of BDNF to the TrkB receptors leads to their dimerization and transphosphorylation on tyrosine residues, allowing the interaction with adaptor proteins and signaling enzymes. TrkB receptors are coupled to the activation of extracellular signal-regulated kinases, members of the mitogen-activated protein kinase family, and of phospholipase $\mathrm{C}-\boldsymbol{\gamma}$ and phosphatidylinositol 3-kinase (PI3-K). Phospholipase C- $\gamma$ generates diacylglycerol and inositol 1,4,5-trisphosphate, and PI3-K leads to the activation of Akt/PKB (Almeida et al., 2005; Reichardt, 2006; Manadas et al., 2007). Each one of the signaling pathways activated by TrkB

Contract grant sponsor: Fundação para a Ciência e Tecnologia; Contract grant numbers: PTDC/SAU-FCF/72283/2006 and SFRH/BD/28139/ 2006.

^Correspondence to: Carlos B. Duarte, Center for Neuroscience and Cell Biology, Department of Zoology, University of Coimbra, 3004-517 Coimbra, Portugal. E-mail: cbduarte@ci.uc.pt

Received 18 March 2008; Revised 5 May 2008; Accepted 5 May 2008

Published online 24 July 2008 in Wiley InterScience (www. interscience.wiley.com). DOI: 10.1002/jnr.21796 
receptors may affect transcription activity (Finkbeiner et al., 1997; Datta et al., 1999; Reichardt, 2006), leading to a complex program of changes in gene expression, as observed in the mouse brain (Koponen et al., 2004), in cultured hippocampal neurons (Ring et al., 2006), and in a neuroblastoma cell line (Schulte et al., 2005). The multiple effects of TrkB receptor on transcription activity partly accounts for the diversity of functional roles of BDNF, including its role in synaptic plasticity (reviewed by Carvalho et al., 2008). The role played by BDNF in numerous neurological disorders (Hashimoto et al., 2005; Chao et al., 2006) may also involve transcription-dependent mechanisms.

Quantitative real-time reverse transcription-polymerase chain reaction (qRT-PCR) is a powerful technique broadly used for mRNA quantification (Dheda et al., 2005; Huggett et al., 2005). Because there are several steps in qRT-PCR that may show variations between genes and samples, such as reverse transcription and the qPCR reaction (Pfaffl, 2001; Ramakers et al., 2003), the results are usually normalized in order to allow accurate comparisons between samples. Several normalization strategies have been proposed, being normalization with internal controls the most widely used (Pfaffl, 2004; Bustin et al., 2005). Endogenous controls, commonly named as reference genes, are subjected to the same conditions as the target genes, and their expression is also measured by qRT-PCR (Suzuki et al., 2000). An ideal reference gene shows an invariable and constant expression among different tissues, independently of the experimental setup. Moreover, the expression level of the endogenous control should preferentially be similar to that of the target genes (Pfaffl, 2004; Sindelka et al., 2006). Although variations in the expression of reference genes may compromise the normalization procedure (Vandesompele et al., 2002; Bustin et al., 2005; Dheda et al., 2005), the commonly accepted reference genes are often used without proper verification of their expression stability under the specific experimental conditions used. For example, $\beta$ actin and glyceraldehyde-3-phosphate-dehydrogenase are frequently used for normalization of gene expression data, although their expression may change with cell proliferation and differentiation (Bustin, 2000; Goidin et al., 2001; Glare et al., 2002; Schmittgen and Zakrajsek, 2000; Zhu and Altmann, 2005; Sindelka et al., 2006, 2007). Therefore, $\beta$-actin and glyceraldehyde-3-phosphate-dehydrogenase expression may be unsuitable as endogenous controls for qRT-PCR, at least for certain experimental protocols. Inaccurate normalization leads in inadequate quantification of mRNA levels and spurious conclusions (de Kok et al., 2005).

The use of endogenous control genes allows for the correction of experimental variations, such as in sample extraction, quantification, and reverse transcription reaction efficiency (Pfaff, 2004; Kubista et al., 2007). The available evidence indicates that there are no universal reference genes (Huggett et al., 2005), and therefore, the endogenous control genes have to be selected for each specific experimental condition (Pfaffl et al., 2004). A reliable and accurate quantification of mRNA expression requires a prior and proper validation of suitable endogenous control genes (Vandesompele et al., 2002; Andersen et al., 2004), and this is particularly relevant in studies addressing the expression of genes that show small changes for the experimental conditions under investigation.

This study aimed at identifying proper endogenous control genes for normalization of gene expression in BDNF-stimulated neurons. For this purpose, a panel of eight potential endogenous control genes was tested regarding their expression in BDNF-stimulated cells. Although several tools to identify the most stably expressed genes in a specific setup have been developed, none is universally accepted. Therefore, the potential endogenous control genes were tested by two different software packages, geNorm (Vandesompele et al., 2002) and NormFinder (Andersen et al., 2004), both available in the GenEx package software (MultiD Analyses, Sweden). Our results show that a careful validation of the reference genes used in the normalization of qRT-PCR data is needed for an accurate quantification of changes in gene expression.

\section{MATERIALS AND METHODS}

\section{Hippocampal Cultures}

Primary cultures of rat hippocampal neurons were prepared from the hippocampi of E18-E19 Wistar rat embryos after treatment with trypsin $(0.06 \%)$ for $15 \mathrm{~min}$ at $37^{\circ} \mathrm{C}$ (Gibco Invitrogen, Paisley, United Kingdom) and deoxyribonuclease I $(5.36 \mathrm{mg} / \mathrm{mL})$, in $\mathrm{Ca}^{2+}$ - and $\mathrm{Mg}^{2+}$-free Hanks' balanced salt solution (HBSS), as previously described (Caldeira et al., 2007). The hippocampi were then washed with HBSS containing $10 \%$ fetal bovine serum (BioWittaker Europe, Belgium) to stop trypsin activity, and transferred to Neurobasal medium (Gibco Invitrogen) supplemented with B27 supplement (1:50 dilution; Gibco Invitrogen), $25 \mu \mathrm{M}$ glutamate, $0.5 \mathrm{mM}$ glutamine, and $0.12 \mathrm{mg} / \mathrm{mL}$ gentamycin. The cells were dissociated in this solution and were plated in six-well plates coated with poly-D-lysine $(0.1 \mathrm{mg} / \mathrm{mL})$. The cultures were maintained in a humidified incubator of $5 \%$ $\mathrm{CO}_{2} / 95 \%$ air at $37^{\circ} \mathrm{C}$ for 7 days. Cultures were stimulated with $100 \mathrm{ng} / \mathrm{mL}$ BDNF (Peprotech, London, United Kingdom) for $30 \mathrm{~min}$ or $3 \mathrm{hr}$.

\section{Total RNA Isolation, RNA Quality, and RNA Concentration}

Total RNA from seven DIV-cultured hippocampal neurons was extracted with TriZol reagent (Invitrogen, Barcelona, Spain), following the manufacturer's specifications. The full content of a six-well cluster plate, with a density of 850,000 cells $/ \mathrm{mL}$, was collected for each experimental condition. After the addition of chloroform and phase separation, the RNA was precipitated by the addition of isopropanol. The precipitated RNA was washed once with $75 \%$ ethanol, centrifuged, air dried, and resuspended in $60 \mu \mathrm{L}$ of RNasefree water (Gibco Invitrogen). The whole procedure was performed at $4^{\circ} \mathrm{C}$. 
RNA quality and integrity was assessed by the Experion automated gel electrophoresis system (Bio-Rad, Amadora, Portugal). A virtual gel was created for each sample, allowing the detection of degradation of the reference markers RNA $18 \mathrm{~S}$ and 28S. Samples showing RNA degradation or contamination by DNA were discarded. RNA concentration was determined by the fluorescent dye Ribogreen (InvitrogenMolecular Probes). The samples were formed into aliquoted and stored at $-80^{\circ} \mathrm{C}$ until further use.

\section{Reverse Transcription}

For first-strand cDNA synthesis, $1 \mu \mathrm{g}$ of total RNA was mixed with Random Hexamer Primer $\mathrm{p}(\mathrm{dN})_{6}$ followed by $10 \mathrm{~min}$ denaturation at $65^{\circ} \mathrm{C}$ to ensure loss of secondary structures that may interfere with the annealing step. The samples were chilled on ice, and the template-primer mix was then supplemented with reaction buffer $(50 \mathrm{mM}$ Tris/ $\mathrm{HCl}, 30 \mathrm{mM}$ $\mathrm{KCl}, 8 \mathrm{mM} \mathrm{MgCl}$, $\mathrm{pH}$ 8.5), Protector RNase Inhibitor (20 $\mathrm{U})$, dNTPs (1 mM each), and finally AMV Reverse Transcriptase (10 U; Roche, Carnaxide, Portugal) in a $20 \mu \mathrm{L}$ final volume. The reaction was performed at $25^{\circ} \mathrm{C}$ for $10 \mathrm{~min}$, followed by $30 \mathrm{~min}$ at $55^{\circ} \mathrm{C}$, for primer annealing to the template and cDNA synthesis, respectively. The reverse transcriptase was then denatured for $5 \mathrm{~min}$ at $85^{\circ} \mathrm{C}$, and the sample was then cooled to $4^{\circ} \mathrm{C}$ for $5 \mathrm{~min}$, and finally stored at $-80^{\circ} \mathrm{C}$ until further use.

\section{Primer Design}

Primers for real-time PCR were designed by Beacon Designer 7 software (Premier Biosoft International, Palo Alto, $\mathrm{CA})$, and the following considerations were taken: 1) GC content about $50 \%$; 2) annealing temperature $\left(T_{\mathrm{a}}\right)$ between $55 \pm 5^{\circ} \mathrm{C}$; 3) secondary structures and primers-dimers were avoided; 4) primer length 18-24 bp; 5) final product length 100-200 bp.

\section{Real-Time PCR}

For gene expression analysis, $2 \mu \mathrm{L}$ of 1:100 diluted cDNA was added to $10 \mu \mathrm{L} 2 \times$ SYBR Green Master Mix (Bio-Rad), and the final concentration of each primer was $250 \mathrm{nM}$ in $20 \mu \mathrm{L}$ total volume. The thermocycling reaction was initiated with activation of Taq DNA polymerase by heating at $95^{\circ} \mathrm{C}$ during $30 \mathrm{sec}$, followed by 45 cycles of a 10 -sec denaturation step at $95^{\circ} \mathrm{C}$, a $30 \mathrm{sec}$ annealing step at $53^{\circ} \mathrm{C}$, and a $30 \mathrm{sec}$ elongation step at $72^{\circ} \mathrm{C}$. The fluorescence was measured after the extension step by the iQ5 Multicolor Real-Time PCR Detection System (Bio-Rad). After the thermocycling reaction, the melting step was performed with slow heating, starting at $55^{\circ} \mathrm{C}$ and with a rate of $0.5^{\circ} \mathrm{C}$ per $10 \mathrm{sec}$, up to $95^{\circ} \mathrm{C}$, with continuous measurement of fluorescence, allowing detection of possible nonspecific products. The assay included a nontemplate control and a standard curve (in 10fold steps) of cDNA for assessing the efficiency of each set of primers. All reactions were run in duplicate to reduce confounding variance.

\section{Data Processing}

The threshold cycle $\left(C_{t}\right)$ represents the detectable fluorescence signal above background resulting from the accumulation of amplified product, and is a proportional measure of the starting target sequence concentration. $C_{t}$ was measured in the exponential phase and therefore was not affected by possible limiting components in the reaction. For every run performed, $C_{t}$ was set at the same fluorescence value.

Data analysis was performed by GenEx (MultiD Analyses) software for real-time PCR expression profiling, which contains both geNorm and NormFinder algorithms. This software enables further determination of the optimal number of endogenous control genes by using the NormFinder algorithm. Because geNorm can only work on linear data, $C_{t}$ values from qRT-PCR were converted into relative quantities by the appropriate tool available in the GenEx software $\left(C_{t}\right.$ values were calculated as a fraction of the maximum value). Where indicated, statistical analysis of the log-transformed expression data was performed by the Student's $t$-test.

\section{RESULTS}

To identify the best internal control genes in BDNF-stimulated hippocampal neurons, the expression of eight commonly used reference genes (Gapdh, glyceraldehyde-3-phosphate dehydrogenase; Actb, b-actin zipcode binding protein 1; Tuba1, tubulin alpha1-A chain; rRNA18S, 18S rRNA gene; Pgk1, phosphoglycerate kinase 1; Tbp, TATA box binding protein; Ppia, peptidylprolyl isomerase A; and Hprt1, hypoxanthine guanine phosphoribosyl transferase I) was evaluated (de Kok et al., 2005; Jung et al., 2007). Table I provides information about the primers and reference and target genes. All reference genes have known functions in the cell, ranging from glycolysis (Gapdh and Pgk1), cytoskeletal protein $(A c t b)$, cell mobility (Tuba1), regulation of transcription (Tbp), protein folding and transport (Ppia), purine metabolism (Hprt1), and protein synthesis (rRNA 18S).

The eight reference genes tested show different expression levels in cultured hippocampal neurons, as determined by the average threshold cycle $\left(C_{t}\right)$ for the selected fluorescence intensity (Fig. 1). rRNA $18 S$ shows the highest expression level, corresponding to an early $C_{t}$ value, whereas $A c t b$ displays the lowest expression levels, with a late $C_{t}$ value. However, none of the genes showed a constant expression on BDNF stimulation, as observed in the scatterplot shown in Figure 1. It was thus inaccurate to randomly pick up a given set of genes and use them as stable reference genes, so we further investigated the expression stability of the selected genes in hippocampal neurons on BDNF stimulation by geNorm (Vandesompele et al., 2002) and NormFinder (Andersen et al., 2004) algorithms. These applications allow checking for the stability of endogenous control genes.

geNorm (Vandesompele et al., 2002) is based in an algorithm that ranks all tested genes according to their stability in every sample tested (control, and samples 


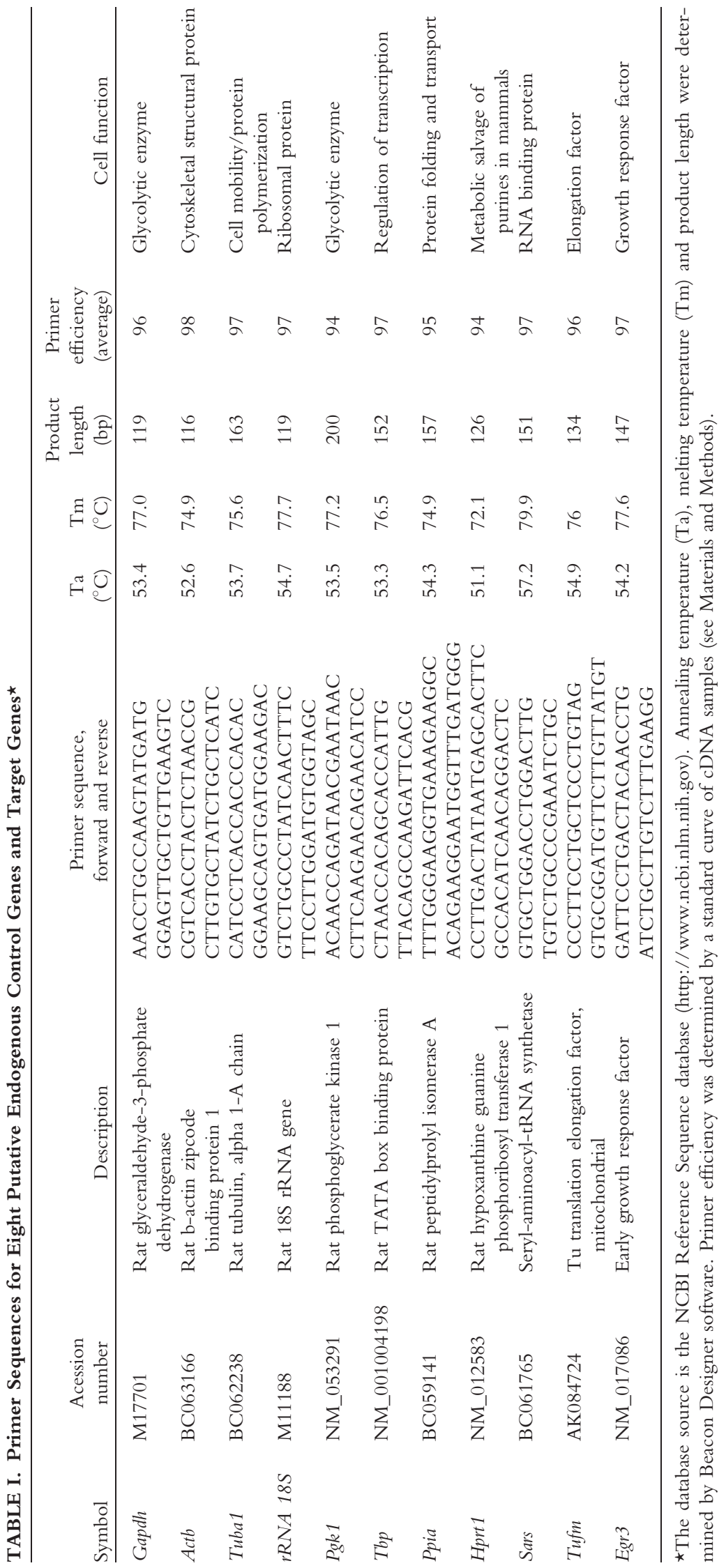




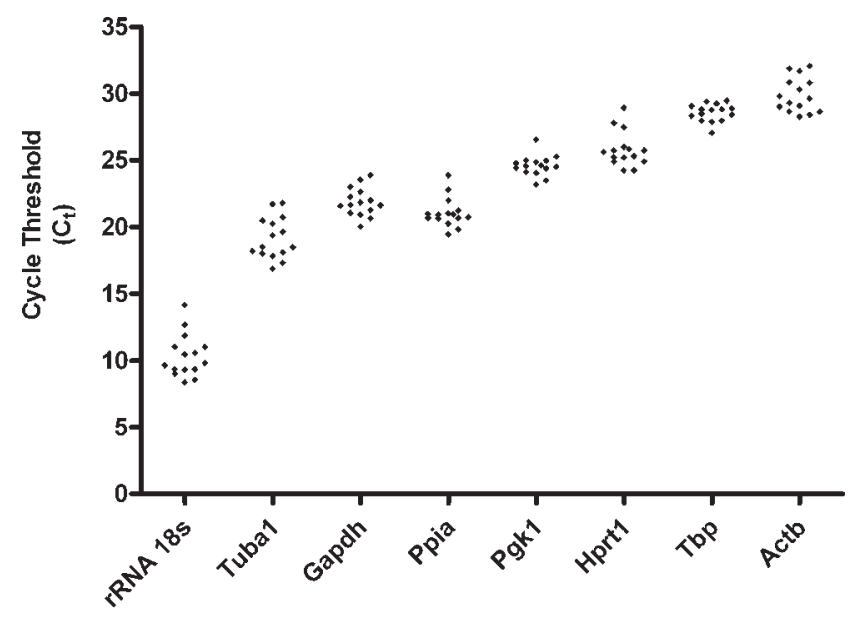

Fig. 1. Expression level $\left(C_{t}\right)$ of eight potential control genes tested in cultured hippocampal neurons. The relative expression values of rRNA 18S, Tuba1, Ppia, Gapdh, Pgk1, Hprt1, Tbp, and Actb was determined under control conditions and in hippocampal neurons stimulated with $100 \mathrm{ng} / \mathrm{mL} \mathrm{BDNF}$ for $30 \mathrm{~min}$ or $3 \mathrm{hr}$. The data points are the results of five independent transcription reactions performed in independent preparations.

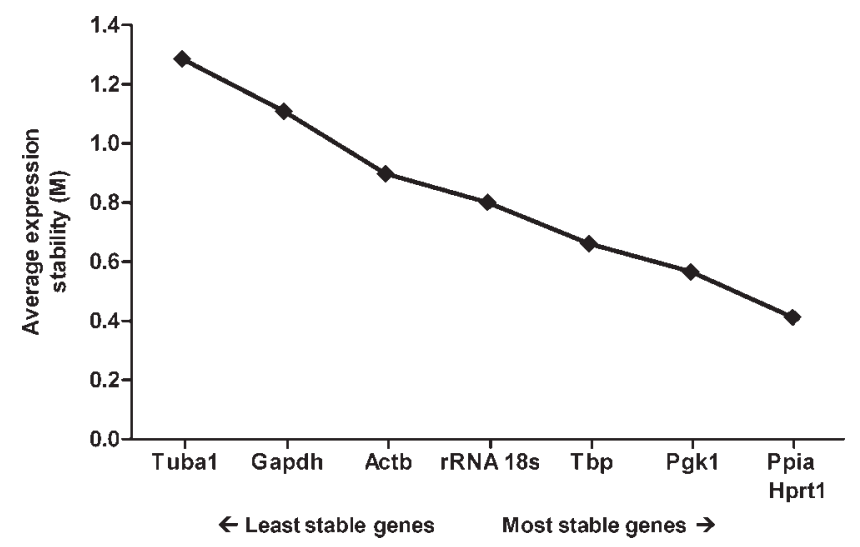

Fig. 2. Average expression stability values $(M)$ during stepwise exclusion of the least stable reference gene in the BDNF-stimulated cells, resulting in the combination of the two most stable reference genes by geNorm application. The highest $M$ values correspond to least stable genes, Tuba1 and Gapdh, while the lowest $M$ values correspond to the most stable genes, Ppia and Hprt1. The raw data analyzed are presented in Figure 1.

treated with BDNF for $30 \mathrm{~min}$ or $3 \mathrm{hr}$ ). This algorithm selects the best two internal control genes for a given experimental setup since it is based on the assumption that if the expression of two genes is constant, its ratio should be constant across all samples. Therefore, this method leads to the selection of gene pairs with similar expression patterns. The algorithm calculates an expression stability value $(M)$ during stepwise exclusion of the least stable control gene, with the highest $M$ values corresponding to the least stable genes, and vice versa. From the list of eight tested genes, geNorm selected Ppia and

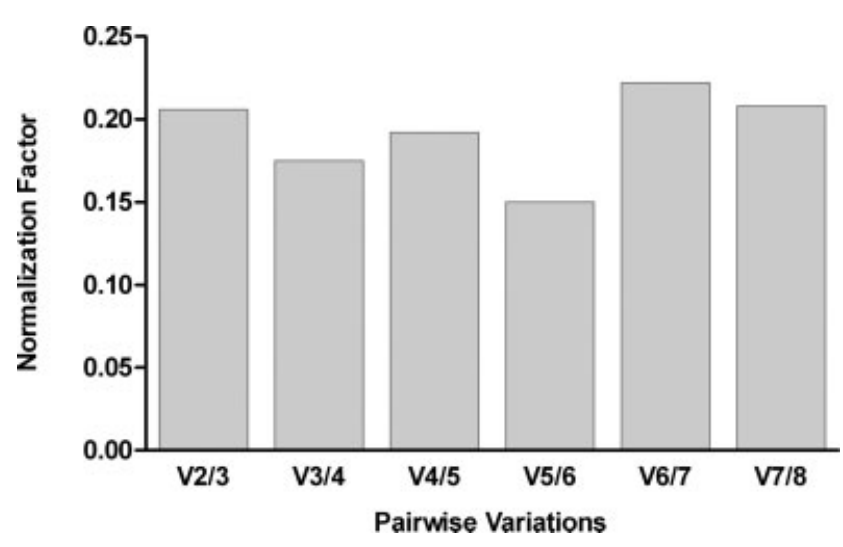

Fig. 3. Determination of the optimal number of reference genes for normalization by pairwise variation $(\mathrm{V} n / n+1)$ between the $\mathrm{NF}_{n}$ and $\mathrm{NF}_{n+1}$ using the geNorm application. V2/3 is the addition of Pgk1; V3/4 is the addition of Tbp; V4/5 is the addition of $r R N A$ $18 S$; V5/6 is the addition of $A c t b ; \mathrm{V} 6 / 7$ is the addition of Gapdh; and $V 7 / 8$ is the addition of Tuba1. The raw data analyzed are presented in Figure 1.

Hprt1 as the most stable genes in samples from control and BDNF-treated hippocampal neurons (Fig. 2); these two genes showed a similar expression pattern in the samples used (Fig. 1). Tuba1 and Gapdh were the most variable genes, corresponding to the highest $M$ values.

The geNorm application also determines the optimal number of internal control genes by using the normalization factor (NF), which is based on the geometric mean of the expression levels of the best endogenous control genes, calculated by the stepwise inclusion of the following best-ranked endogenous gene (Vandesompele et al., 2002). The NF is a useful tool for the determination of the optimal number of control genes because the effect of the additional gene will be reflected in the NF. In this analysis, the number of control genes necessary for an accurate normalization process is achieved when the addition of a new gene does not greatly influence the NF. Figure 3 shows the multiple NF values calculated from the stepwise inclusion of the best ranked gene. The addition of the third best gene-Pgk1-raised the NF to 0.21 (V2/3), and the addition of the fourth best control gene, Tbp, reduced the NF (V3/4 = 0.18). Since Tbp did not greatly affect NF, it can be excluded from the normalization process because its addition will not improve the analysis. Addition of the most variable genes, Tuba1 and Gapdh, increased the NF (V6/7 = 0.22 and $\mathrm{V} 7 / 8=0.21)$, reflecting their instability.

Because there is no consensus about the most appropriate tools for the validation of the best internal control genes in a specific experimental condition, we also validated the panel of eight genes by the NormFinder algorithm (Andersen et al., 2004). This approach examines the expression stability of each gene independently of other genes and tests combinations of gene pairs to compensate for the variability of the system. The NormFinder application estimates the variation in gene 


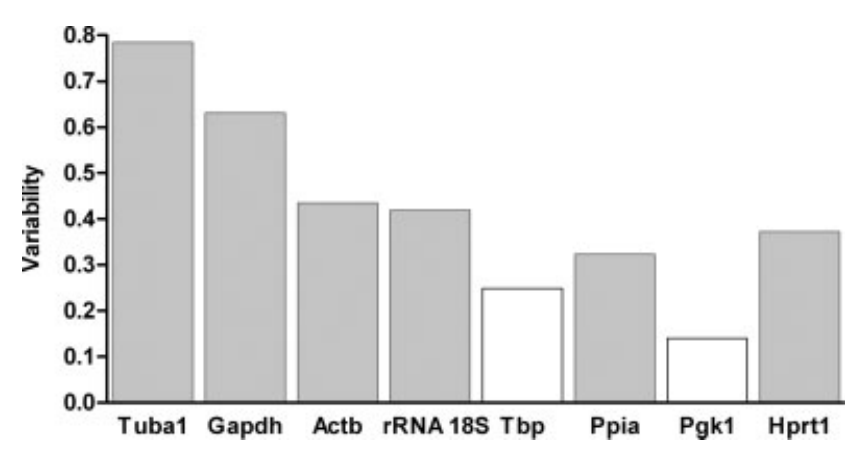

Fig. 4. Ranking of stability of reference gene expression, as determined by NormFinder. The variability of the tested control genes was calculated in samples from hippocampal neurons stimulated (30 min or $3 \mathrm{hr}$ ) or not with $100 \mathrm{ng} / \mathrm{mL}$ BDNF. The algorithm selected $P g k 1$ as the most stable gene and Pgk1 and Tbp as the most stable gene pair, which shows the minimal inter- and intragroup variation. The raw data analyzed are presented in Figure 1.

expression, taking into account and avoiding variation across subgroups. The stability value calculated by NormFinder is a direct measure of expression variation, allowing us to evaluate the systemic error introduced when the selected genes are used for normalization (Andersen et al., 2004). The main difference between NormFinder and geNorm is that NormFinder selects the best gene taking into account the experimental conditions used; it also selects the best combination of gene pair in order to compensate for fluctuation in the experimental data. The variability of each one of the eight genes tested is shown in Figure 4. NormFinder selected Pgk1 as the gene that showed smaller intergroup variation, indicating that $P g k 1$ displayed the least variability in experiments testing the effect of BDNF in cultured hippocampal neurons. Tbp expression also showed low variability as determined with NormFinder, and therefore, the combination of Tbp and Pgk1 is recommended by this algorithm to compensate for fluctuation of experimental data on changes in gene expression in response to BDNF stimulation. The NormFinder algorithm available in the GenEx package (MultiD Analysis) also allows for the determination of the optimal number of control genes to be used in the normalization processes, through the calculation of the accumulated standard deviation (Acc. SD). The Acc. SD of the eight potential genes tested is shown in Figure 5. The optimal number of endogenous control genes is indicated by the minimal value for the accumulated standard deviation, 0.31, achieved when the six endogenous control genes that show greater stability are used.

Comparison of the ranking of expression stability for the eight reference genes tested in hippocampal neurons subjected to stimulation with BDNF, as determined by the geNorm (Fig. 2) and NormFinder (Fig. 4) algorithms, shows that the most appropriate pair of reference genes identified by each of the methods is distinct (Ppia, Hprt1; Pgk1, Tbp for geNorm and NormFinder, respectively). However, in both cases, the most variable genes

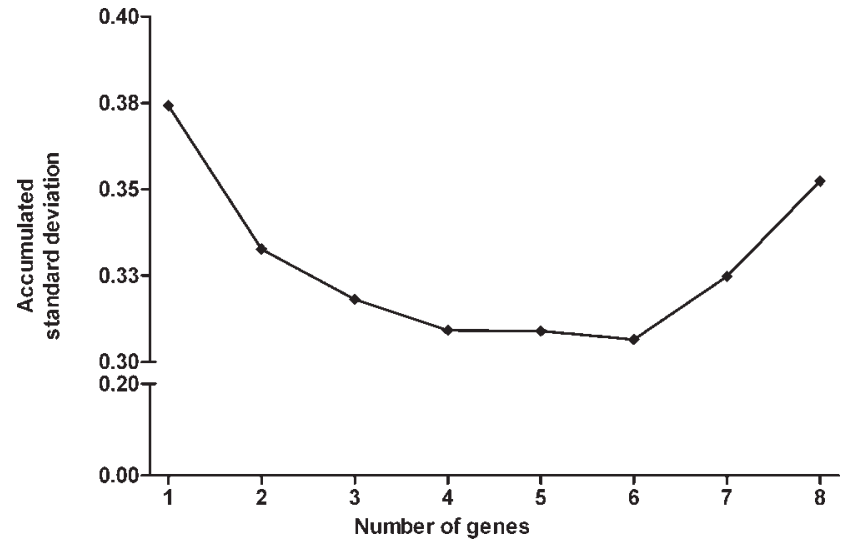

Fig. 5. Determination of the optimal number of reference genes for normalization based on the calculation of the Acc. SD. Data analysis was performed by NormFinder, an application available in the GenEx software. The lowest value for the Acc. SD was achieved when using the best six ranked reference genes (Acc. SD $=0.31$ ). The raw data analyzed are presented in Figure 1.

were those that are more often used as reference genes (Tuba1, Gapdh, and Actb).

We have compared two sets of genes, Tuba1/ Gapdh/Actb (least stable genes) and Pgk1/Tbp/Ppia/Hprt1 (most stable genes), for normalization of BDNF-induced changes in gene expression in cultured hippocampal neurons. The two sets of genes were grouped on the basis of their stability in BDNF-stimulated hippocampal neurons (see above). Figure 6 shows the effect of BDNF on the expression of two genes coding for proteins belonging to the translation machinery, seryl-aminoacyltRNA synthetase (Sars) and Tu mitochondrial translation factor (Tufm), and early growth response 3 (Egr3). Sars is an RNA binding protein responsible for the catalytic transfer of L-serine to tRNA and Tufm is involved in the chain formation during polypeptide synthesis at the ribosome. When normalization was performed with the genes showing the least variability (Pgk1, Tbp, Ppia and Hprt1; Fig. 6A) statistically significant effects $(P<0.05)$ of BDNF were found on the expression of Tufm ( $P=$ $0.020)$ and $\operatorname{Egr} 3(P=0.012)$, with the former gene showing a decrease in expression level and the latter being upregulated. The effect of BDNF on the expression of Egr3 in cultured hippocampal neurons is in agreement with a previous report (Roberts et al., 2006). In contrast, no significant effects of BDNF on the expression of Sars, Tufm or Egr3 were found when normalization was performed with the genes showing higher variability (Fig. 6D). Although both normalization procedures showed an upregulation of Sars in BDNFtreated hippocampal neurons, the differences were not statistically significant. Interestingly, normalization with the most stable set of genes showed a downregulation of Tufm expression, in contrast to the slight increase in expression observed when the least stable genes were used. 

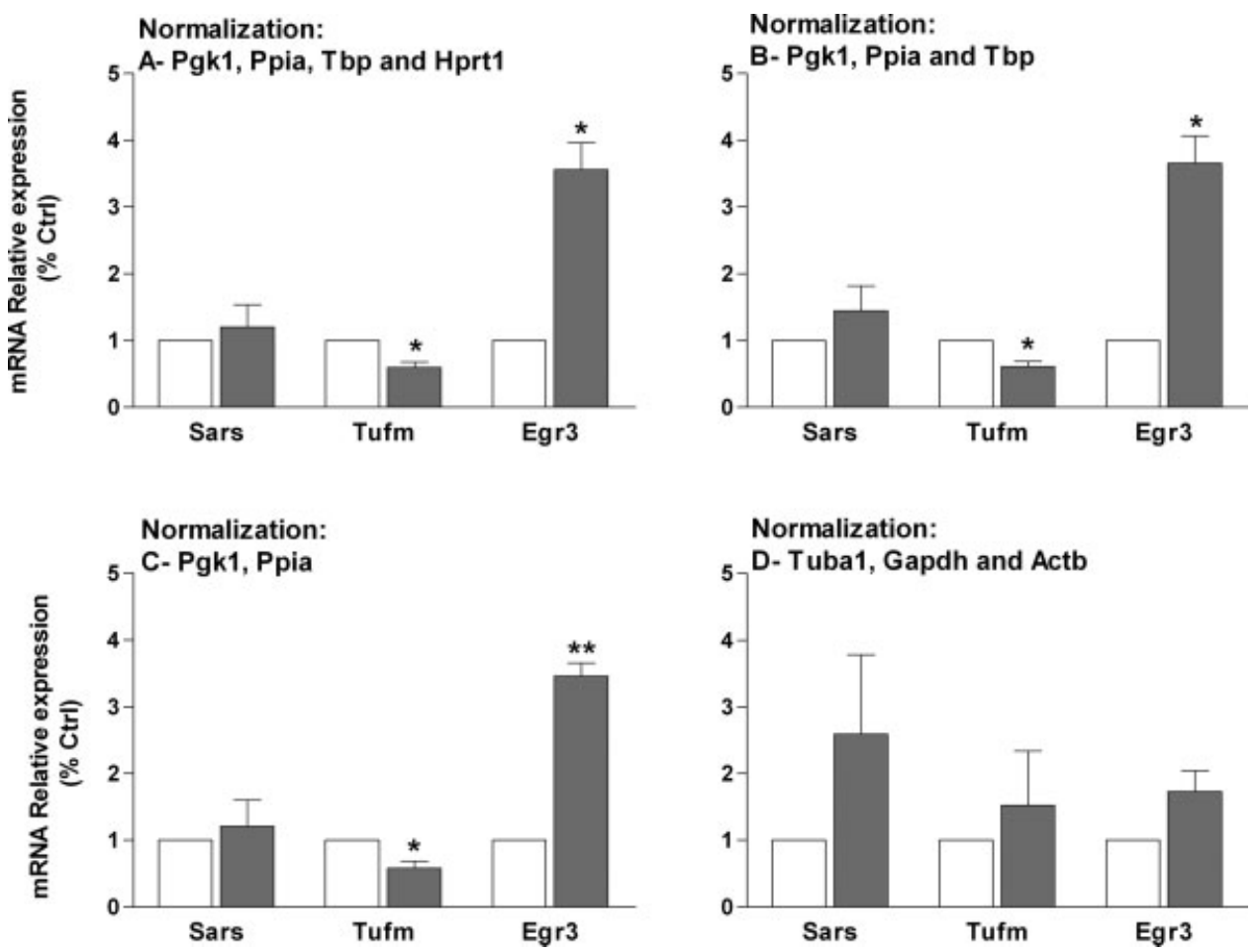

Fig. 6. Effect of BDNF on the expression of Sars, Tufm, and Egr3, as influenced by the sets of genes used for normalization. Hippocampal neurons were stimulated or not with $100 \mathrm{ng} / \mathrm{mL}$ BDNF for $3 \mathrm{hr}$. Normalization with the four (A: Pgk1, Tbp, Ppia, and Hprt1), three (B: Pgk1, Tbp and Ppia) and two (C: Pgk1 and Ppia) best ranked control genes in BDNF stimulated hippocampal neurons are shown. Normalization with the worst scoring control genes Tuba1, Gapdh,

To determine how the number of reference genes used in the normalization process affects the calculated BDNF-induced changes in gene expression, we further analyzed the results using two or three genes in the normalization. The use of the three most stable genes ( $P g k 1$, Tbp, Ppia; Fig. 6B) in the normalization gave results similar to those obtained when four genes were used (Fig. 6A). In contrast, when two genes were used for normalization (Ppia and Pgk1; Fig. 6C) the effect of BDNF on the expression level of Egr3 was even more significant $(P$ $=0.009)$. The effect of BDNF on both Sars and Tufm expression levels was not changed when Ppia and Pgk1 were used as reference genes.

\section{DISCUSSION}

Reference genes are frequently used to normalize qRT-PCR results (Vandesompele et al., 2002; Ayers et al., 2007). Their use as normalizer genes has been accepted as they are thought to be constitutively expressed and invariable. However, several reports have shown that commonly used reference genes can indeed be differentially expressed among different tissues, in different developmental stages, and in distinct experimental

and $A c t b$ is shown in panel $\mathbf{D}$. The average $C_{t}$ values obtained for Sars, Tufm, and Egr3 were 24.12 $\pm 1.32,25.69 \pm 0.53$, and $30.76 \pm$ 2.32 , respectively. The results are the average \pm SEM of four independent transcription reactions, performed in independent preparations. ${ }^{\star} P<0.05,{ }^{\star} \star P<0.01$ as determined by Student's $t$-test performed on log-transformed expression data.

conditions (Thellin et al., 1999; Suzuki et al., 2000; Glare et al., 2002; Sindelka et al., 2006, 2007). In the present study, we tested the expression stability of a panel of eight potential endogenous control genes in control and BDNF-stimulated hippocampal neurons. The genes tested are constitutively expressed in the cells and have independent functions. Although the geNorm and NormFinder algorithms provided distinct results concerning the most stably expressed genes in BDNFtreated hippocampal neurons (Ppia, Hprt1; Pgk1, Tbp, respectively), both showed a higher variability in the expression of genes often used as control genes (Tuba1, Gapdh, and Actb). These results show that a careful selection of the genes used for normalization is required for each experimental protocol. Because different methods of analysis of gene expression provided distinct results concerning the most stably expressed genes in cultured hippocampal neurons subjected to stimulation with BDNF (Figs. 3 and 5), a combination of at least three genes from the group-Ppia, Pgk1, Hprt1, and Tbpshould be used as a control (however, see below for further discussion).

Several tools, including geNorm (Vandesompele et al., 2002) and NormFinder (Andersen et al., 2004), have been developed to identify the most stable genes. 
In the present work, we found that the use of the two algorithms provided different results concerning the most stable genes in cultured hippocampal neurons subjected to stimulation with BDNF. Similar discrepancies were also found in the selection of control genes for relative quantification of gene expression during differentiation of human embryonic stem (ES) cells, as well as in differentiated mouse ES cells and in developing mouse embryo (Willems et al., 2006). These differences between the results obtained with the geNorm and NormFinder algorithms may be explained on the basis of the distinct mathematical approaches used. geNorm tends to select the pair of genes with the highest degree of similarity in the expression pattern, in contrast to NormFinder that ranks first the genes with minimal inter- and intragroup variation (Andersen et al., 2004). This renders geNorm more sensitive to coregulated genes than NormFinder, which analyses the expression stability of each gene independently.

geNorm is based in the assumption that the expression ratio of two ideal reference genes is constant across every sample (Vandesompele et al., 2002), ranking the genes on the basis of their expression-stability value, $M$, with highest $M$ value corresponding to less stable genes. By using samples from control and BDNF-treated hippocampal neurons, we found that the most stable genes, corresponding to the lowest $M$ value, were Ppia and Hprt1. Interestingly, Tuba1, Gapdh, and Actb, which are widely used as reference genes, were ranked as the least stable control genes in BDNF-stimulated cells. This algorithm also calculates the NF value, which indicates the optimal number of control genes to be used, and cutoff values of 0.20 (Ayers et al., 2007) to 0.15 (Vandesompele et al., 2002; Spinsanti et al., 2006) have been suggested. Because there is no standard value for NF, our results suggest that the use of the three most stable genes (Ppia, Hprt1, and Pgk1) is sufficient for an accurate normalization process in studies regarding the BDNFinduced changes in gene expression. It is interesting to note that the expression of Gapdh and Pgk1, two genes coding for glycolytic enzymes, show distinct patterns of regulation in response to BDNF stimulation, Pgk1 being more stable.

In this work, we also used NormFinder (Andersen et al., 2004) to validate the putative control genes in BDNF-stimulated cultured hippocampal neurons. By using this algorithm, we identified Pgk1 as the most stable gene and Pgk1-Tbp as the best combination of genes, showing the least variation across the subgroups. Furthermore, Tuba1 and Gapdh, two commonly used reference genes, showed the least expression stability of all control genes tested, in agreement with the results obtained with the geNorm (Fig. 2). Therefore, the use of Tuba1 and Gapdh as reference genes in studies on the regulation of gene expression by BDNF in hippocampal neurons should be avoided. With the NormFinder approach, we calculated the Acc. SD of the tested genes, and we found that the use of the six more stable endogenous control genes is recommended by this program for the normalization processes. However, there was a minor difference between using three $(\mathrm{SD}=0.32)$ or six $(\mathrm{SD}=0.31)$ endogenous control genes, indicating that the three more stable genes calculated by NormFinder can safely be used in the normalization process. The use of seven and eight endogenous control genes (Gapdh and Tuba1) would not improve the normalization, as the Acc. SD increases when these genes are taken into account in the calculation of the Acc. SD.

When we compared the results from the pairwise variations graph and Acc. SD graph, both geNorm and NormFinder gave the same results, with the minimal value for the NF achieved when the six best-ranked genes were included, and the Acc. SD was also minimal when the six more stable genes were considered for normalization. The minimum number of endogenous control genes to be used for normalization in gene expression studies is still under debate; it is necessary to balance the need of using multiple control genes with economical and practical considerations.

The importance of a valid and proper normalization process is clearly shown by the results of Figure 6 , where the normalization of the BDNF-induced changes in gene expression was performed by using four different sets of genes. The conclusions that can be drawn about the effects of BDNF in hippocampal neurons depend on how accurate the normalization process is, especially when dealing with small changes in gene expression. Taken together, the results obtained by geNorm and NormFinder and the tests that used three target genes indicate that three reference genes can safely be used for normalization, leading to accurate and reliable results. Surprisingly, normalization with only two reference genes slightly increased the statistical significance of the BDNF-induced changes in Egr3 expression. However, caution should be used whenever normalizing results with only two reference genes.

In conclusion, our results point out the importance of prior verification of the expression stability of the endogenous control genes in each specific setup and the need of using multiple valid endogenous control genes for an accurate normalization of the results. This is particularly relevant when analyzing cellular responses to agents, such as neurotrophins, that cause multiple changes in gene expression.

\section{ACKNOWLEDGMENTS}

We thank Elisabete Carvalho for her assistance in preparing the cell cultures and Eduardo Lopes (Bio-Rad, Portugal) for helping us set up the real-time PCR methodologies. We also appreciate the help of Anders Bergkvist (MultiD Analyses, Sweden).

\section{REFERENCES}

Almeida RD, Manadas BJ, Melo CV, Gomes JR, Mendes CS, Graos MM, Carvalho RF, Carvalho AP, Duarte CB. 2005. Neuroprotection by BDNF against glutamate-induced apoptotic cell death is mediated by ERK and PI3-kinase pathways. Cell Death Differ 12:1329-1343. 
Althaus HH, Richter-Landsberg C. 2000. Glial cells as targets and producers of neurotrophins. Int Rev Cytol 197:203-277.

Andersen CL, Jensen JL, Orntoft TF. 2004. Normalization of real-time quantitative reverse transcription-PCR data: a model-based variance estimation approach to identify genes suited for normalization, applied to bladder and colon cancer data sets. Cancer Res 64:5245-5250.

Ayers D, Clements DN, Salway F, Day PJ. 2007. Expression stability of commonly used reference genes in canine articular connective tissues. BMC Vet Res 3:7.

Bustin SA. 2000. Absolute quantification of mRNA using real-time reverse transcription polymerase chain reaction assays. J Mol Endocrinol 25:169-193.

Bustin SA, Benes V, Nolan T, Pfaffl MW. 2005. Quantitative real-time RT-PCR—a perspective. J Mol Endocrinol 34:597-601.

Caldeira MV, Melo CV, Pereira DB, Carvalho R, Correia SS, Backos DS, Carvalho AL, Esteban JA, Duarte CB. 2007. Brain-derived neurotrophic factor regulates the expression and synaptic delivery of alphaamino-3-hydroxy-5-methyl-4-isoxazole propionic acid receptor subunits in hippocampal neurons. J Biol Chem 282:12619-12628.

Carvalho AL, Caldeira MV, Santos SD, Duarte CB. 2008. Role of the brain-derived neurotrophic factor at glutamatergic synapses. Br J Pharmacol 153(Suppl 1):S310-S324.

Chao MV. 2003. Neurotrophins and their receptors: a convergence point for many signalling pathways. Nat Rev Neurosci 4:299-309.

Chao MV, Rajagopal R, Lee FS. 2006. Neurotrophin signalling in health and disease. Clin Sci (Lond) 110:167-173.

Datta SR, Brunet A, Greenberg ME. 1999. Cellular survival: a play in three Akts. Genes Dev 13:2905-2927.

de Kok JB, Roelofs RW, Giesendorf BA, Pennings JL, Waas ET, Feuth T, Swinkels DW, Span PN. 2005. Normalization of gene expression measurements in tumor tissues: comparison of 13 endogenous control genes. Lab Invest 85:154-159.

Dheda K, Huggett JF, Chang JS, Kim LU, Bustin SA, Johnson MA, Rook GA, Zumla A. 2005. The implications of using an inappropriate reference gene for real-time reverse transcription PCR data normalization. Anal Biochem 344:141-143.

Finkbeiner S, Tavazoie SF, Maloratsky A, Jacobs KM, Harris KM, Greenberg ME. 1997. CREB: a major mediator of neuronal neurotrophin responses. Neuron 19:1031-1047.

Glare EM, Divjak M, Bailey MJ, Walters EH. 2002. beta-Actin and GAPDH housekeeping gene expression in asthmatic airways is variable and not suitable for normalising mRNA levels. Thorax 57:765-770.

Goidin D, Mamessier A, Staquet MJ, Schmitt D, Berthier-Vergnes O. 2001. Ribosomal 18S RNA prevails over glyceraldehyde-3-phosphate dehydrogenase and beta-actin genes as internal standard for quantitative comparison of mRNA levels in invasive and noninvasive human melanoma cell subpopulations. Anal Biochem 295:17-21.

Hashimoto T, Bergen SE, Nguyen QL, Xu B, Monteggia LM, Pierri JN, Sun Z, Sampson AR, Lewis DA. 2005. Relationship of brainderived neurotrophic factor and its receptor TrkB to altered inhibitory prefrontal circuitry in schizophrenia. J Neurosci 25:372-383.

Huang EJ, Reichardt LF. 2001. Neurotrophins: roles in neuronal development and function. Annu Rev Neurosci 24:677-736.

Huggett J, Dheda K, Bustin S, Zumla A. 2005. Real-time RT-PCR normalisation; strategies and considerations. Genes Immun 6:279-284.

Jung M, Ramankulov A, Roigas J, Johannsen M, Ringsdorf M, Kristiansen G, Jung K. 2007. In search of suitable reference genes for gene expression studies of human renal cell carcinoma by real-time PCR. BMC Mol Biol 8:47.

Koponen E, Lakso M, Castren E. 2004. Overexpression of the full-length neurotrophin receptor trkB regulates the expression of plasticity-related genes in mouse brain. Brain Res Mol Brain Res 130:81-94.
Kubista M, Sindelka R, Tichopad A, Bergkvist A, Lindh D, Forootan A. 2007. The prime technique: real-time PCR data analysis. GIT Lab J 910:33-35.

Manadas BM, Melo CV, Gomes JR, Duarte CB. 2007. Neurotrophin signaling and cell survival. In: Malva JO, Rego AC, Cunha RA, Oliveira $C R$, editors. Interaction between Neurons and Glia in Aging and Disease. Berlin: Springer-Verlag, p 137-172.

Pfaffl MW. 2001. A new mathematical model for relative quantification in real-time RT-PCR. Nucleic Acids Res 29:e45.

Pfaffl MW. 2004. Quantification strategies in real-time PCR. In: Bustin S, editor. A-Z of Quantitative PCR. La Jolla, CA: International University Line.

Pfaffl MW, Tichopad A, Prgomet C, Neuvians TP. 2004. Determination of stable housekeeping genes, differentially regulated target genes and sample integrity: BestKeeper-Excel-based tool using pair-wise correlations. Biotechnol Lett 26:509-515.

Ramakers C, Ruijter JM, Deprez RH, Moorman AF. 2003. Assumption-free analysis of quantitative real-time polymerase chain reaction (PCR) data. Neurosci Lett 339:62-66.

Reichardt LF. 2006. Neurotrophin-regulated signalling pathways. Philos Trans R Soc Lond B Biol Sci 361(1473):1545-1564.

Ring RH, Alder J, Fennell M, Kouranova E, Black IB, Thakker-Varia S. 2006. Transcriptional profiling of brain-derived-neurotrophic factorinduced neuronal plasticity: a novel role for nociceptin in hippocampal neurite outgrowth. J Neurobiol 66:361-377.

Roberts DS, Hu Y, Lund IV, Brooks-Kayal AR, Russek SJ. 2006. Brain-derived neurotrophic factor (BDNF)-induced synthesis of early growth response factor 3 (Egr3) controls the levels of type A GABA receptor alpha 4 subunits in hippocampal neurons. J Biol Chem 281:29431-29435.

Schmittgen TD, Zakrajsek BA. 2000. Effect of experimental treatment on housekeeping gene expression: validation by real-time, quantitative RT-PCR. J Biochem Biophys Methods 46:69-81.

Schulte JH, Schramm A, Klein-Hitpass L, Klenk M, Wessels H, Hauffa BP, Eils J, Eils R, Brodeur GM, Schweigerer L, Havers W, Eggert A. 2005. Microarray analysis reveals differential gene expression patterns and regulation of single target genes contributing to the opposing phenotype of TrkA- and TrkB-expressing neuroblastomas. Oncogene 24:165-177.

Schweigreiter R. 2006. The dual nature of neurotrophins. BioEssays 28:583-594.

Sindelka R, Ferjentsik Z, Jonak J. 2006. Developmental expression profiles of Xenopus laevis reference genes. Dev Dyn 235:754-758.

Sindelka R, Jonak J, Hands R, Bustin SA, Kubista M. 2007. Intracellular expression profiles measured by real-time PCR tomography in the Xenopus laevis oocyte. Nucleic Acids Res 36:387-392.

Spinsanti G, Panti C, Lazzeri E, Marsili L, Casini S, Frati F, Fossi CM. 2006. Selection of reference genes for quantitative RT-PCR studies in striped dolphin (Stenella coeruleoalba) skin biopsies. BMC Mol Biol 7:32.

Suzuki T, Higgins PJ, Crawford DR. 2000. Control selection for RNA quantitation. Biotechniques 29:332-337.

Thellin O, Zorzi W, Lakaye B, De Borman B, Coumans B, Hennen G, Grisar T, Igout A, Heinen E. 1999. Housekeeping genes as internal standards: use and limits. J Biotechnol 75:291-295.

Vandesompele J, De Preter K, Pattyn F, Poppe B, Van Roy N, De Paepe A, Speleman F. 2002. Accurate normalization of real-time quantitative RT-PCR data by geometric averaging of multiple internal control genes. Genome Biol 3:RESEARCH0034.

Willems E, Mateizel I, Kemp C, Cauffman G, Sermon K, Leyns L. 2006. Selection of reference genes in mouse embryos and in differentiating human and mouse ES cells. Int J Dev Biol 50:627-635.

Zhu LJ, Altmann SW. 2005. mRNA and 18S-RNA coapplicationreverse transcription for quantitative gene expression analysis. Anal Biochem 345:102-109. 